\title{
Extended Vertical Handoff Decision in Next Generation Wireless Networks
}

\author{
Shelej Khera ${ }^{1}$, Dr. S. V. A. V Prasad ${ }^{2}$ \\ Research Scholar-ECE Deptt., Lingaya's University, Faridabad, Haryana, India ${ }^{l}$ \\ Professor-ECE Dept, Lingaya's University, Faridabad, Haryana, India ${ }^{2}$
}

\begin{abstract}
In the vision of $4 G$ wireless communications, it is requisite to provide seamless mobility support across heterogeneous access networks. The benefit of heterogeneous network is that, devices can connect to different wireless networks without interruption of service while the network is being switched. The problem in heterogeneous environments is that transition between diverse types of access technologies can lead to reduced performance, mainly due to incompatibility problems. Seamless mobility can be achieved by enabling mobile terminals to conduct seamless handovers across diverse access networks, that is, seamlessly transfer and continue their ongoing sessions from one access network to another. One major challenge in cellular/WLAN internetworking is how to take advantage of the wide coverage and almost universal roaming support of cellular networks and the high data rates of WLAN. Many issues should be carefully addressed to achieve seamless interworking, such as mobility management, resource allocation, call admission control, security and billing. The vertical handoff requirements are potential of the network, network cost, handoff latency, network conditions, power consumption and user's preference. These must be considered during vertical handoff. In this research paper a new vertical handoff decision based on ARM \& AI Techniques is designed. A refined, intelligent Associative Rule Mining (ARM) and Artificial Intelligence (AI) based technique is used to execute the vertical handoff intelligent mechanism in fourth generation wireless networks to produce an effective service which reduces the handoff delay and complexity.
\end{abstract}

Keywords: $4 G$, Vertical Handover decision (VHD), ARM, AI

\section{Introduction}

Wireless networks are fetching more significant and accepted method to provide world wide information access to users on travel. A movable device through alternative wireless subnets has many means of accessing the wired infrastructure with several wireless network interfaces

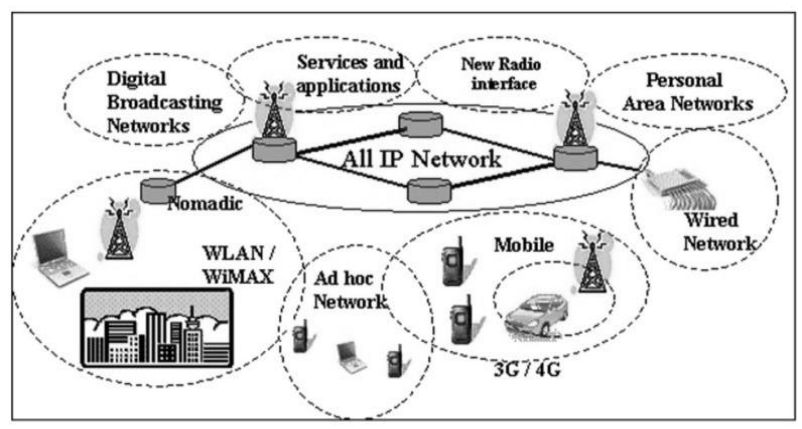

Figure: Types of Network

A subscriber may move from his office, where his laptop or personal digital device (PDD) is connected to infrared network present in room, to somewhere else in the edifice, where it is linked to a network in building having wide radio frequency (RF) network. As the same subscriber moves outside, then he is connected to broad area network, and then to any other building that has been connected through any other building-wide RF network. The grouping of interfaces of wireless network, in building, spanning in room, campus, large area and regional cell sizes, which fit in a group of network interfaces, is called overlay network structure of wireless.

\section{Need of Handoff}

Following are the major reasons for the need of handoff.

a. The person on decision is traveling and moving in one cell and catching the area of second area cell, the decision shouldn't disconnect the call when he shifts to another cell. 
b. Every cell has its own capacity. Once the capability of connecting new decisions for present cell is employed and present or new call from a mobile that is found within a neighborhood crossed by another cell is transferred to it, it should have some capability within the initial cell for alternative users.

c. Due to the interference of two phones present in the different cells of the same channels.

d. The management of the quick and slow moving users consistent with their demand to avoid the potential interference to the opposite users in cells.

e. For scaling back the interference of a small neighboring cell because of the "near-far" impact if the mobile phone has still a superb affiliation to its present cell.

\section{Overview Of Vertical Handoff Process}

It is the process in which a mobile terminal changes from its present connection point to another access network. It is method of keeping user's active communication. This consists of various stages like: Initiation of handover, decision of handover and Execution of handover.

- Initiation of Handover: In this step selection of target point of connection is considered, depending upon certain parameters and the time of the handover

- Handover Decision: The new point of attachment is decided and created in this process.

- Handover Execution: New channel is allotted in this process. The best network is connected by VHD algorithms among all the available channels

\section{Vertical Handoff Decision (VHD) Criteria}

Various parameters are considered in vertical handoff decision algorithms. These are explained as follows:

i. Strength of Received Signal: It is main parameter for decision in vertical handoff. Received signal strength is measured easily and it directly relates to service quality. Horizontal handover algorithms mostly use this parameter

ii. Network connection time: It is the most essential parameter. This time means the extent that user has been connected to the current position of connection

iii. Bandwidth Available: Available bandwidth is the calculation of existing information and communication resources.

iv. Consumption of Power: If the battery of mobile terminal is low the consumption of power becomes main issue

v. Monetary cost: As every network has its own charging policies, the charges of network service must also be considered in implementing handover decisions

vi. Security: When integrity of the transmitted data is vital, systems having advanced level of security should be selected over other one that offers lower level of security of data.

vii. Preferences of User: Individual user's choice can lead to the selection of one type of network over the other networks

\section{RSS Based VHD Algorithms:}

\section{Classification Of VHD Algorithm:}

Received Signal Strength is the most important criteria in the RSS based algorithms. Vertical handoff decision algorithms assess the received signal strength of the present connection against the others to make handover decisions.

\section{VHD Algorithms Based on Bandwidth:}

This algorithm uses available bandwidth as the main criteria for the handover. Remaining bandwidth and requirements of user are considered in taking decision whether to switch from wireless local area network to Wireless Wide Area Network (WWAN) and vice versa. Location of movable unit is also taken in to consideration. As available bandwidth is considered as main criteria, it gives good throughput for vertical handoff decision. Lower handover latency is achieved for delay-sensitive applications.

\section{VHD Algorithms Based on Cost Function:}

Group of networks and other parameters such as strength of received signal, available bandwidth, service cost, network covering area, security, battery power are chosen in these types of decision algorithms for estimating the work quality of desired networks. Handoff decision is made accordingly. According to priority, cost of each possible target network is calculated in this method. The use of cost function makes this method useful. Handover blocking probability is reduced and percentage of user fulfilled requests is increased. In these decision algorithms the normalization and weights distribution methods are given in the system. The highest weight function is chosen as the target for switching. Advantages of this type of algorithm are reduced decision delay of handoff, high throughput and reduced blocking rate of handover 


\section{Hybrid Algorithm:}

These algorithms use artificial neural networks (ANN) and fuzzy logic. The parameters of present wireless networks are collected by mobile devices and are sent to a middleware known as vertical handover manager by the obtainable connections. Network handling device, feature collector and ANN training/selector are its three main components. A multilayer feed forward ANN selects the best handover desired wireless network available to mobile terminal based on the preferences users. Superfluous handovers and ping-pong effect is avoided in this algorithm.

\section{Methodology}

Many research works were developed in this field of handoff into networks using wireless technology. But existing methods have the drawbacks that these techniques do not concentrate on the most important parameters in the networks during the handoff. In some research methods the nearest base station congestion has not been considered during transfer of call to the nearest base station. A number of research works reduce these drawbacks by considering the base stations load value during the handover, but those methods do not consider the time of the handover. The quality of service drops below a sufficient level and connection is dropped if handover does not happen at right time. To prioritize calls based on the FIFO scheme the queuing method has been introduced in handover. In these queuing schemes when obtained signal strength of the base station controller in the present cell reaches to certain defined verge the call is queued for servicing a nearby cell. A new call request is given to a channel if there is empty queue and there is at least a free channel in the base station controller. This queuing method also has the disadvantage that when the call achieves the receiver level and then free channel is not available, the connection is not matured i.e. the importance of the call is not taken care of. So the necessity is to develop a new handover technique in the mobile network. All the aforesaid drawbacks presented in the existing techniques have motivated to do the research in this area.

\section{Objectives And Challenges}

There are various problems in the handoff initiation and decision making process. The main technical aim and challenge in obtaining these objectives include in making of a flawless handoff system in various networks are:

a. Production of the Historical information based on the Network arrangement.

b. To handoff the Mobile Nodes using Artificial Intelligence based on historical information.

c. To realize Rule Mining and generate the handoff factor, handoff rules based on Bandwidth, RSS and Cost in terms of time.

d. To select the optimal target network using efficient decision making algorithm.

e. To perform the necessary handoffs in Vertical Communication.

f. Power Savings: minimizes the consumption of power by maintaining the simple vertical handoff decision.

g. Overhead of Bandwidth: To reduce the extra traffic in network used for implementing switching by obtaining optimal network to reduce the complication.

h. Calculation of the improvement obtained by handing off to a particular network. an Extended historical vertical handoff decision function is developed

\section{Mean Handoff Computation Algorithm}

For solving the problem step by step Algorithm is used which can be implemented with help of programming.

1. Start

2. The Input Parameters BW, Time and RSS (Signal Strength) are setup

3. The Fuzzy Inference System based on Input parameters with Membership Functions is setup.

4. The Membership Functions value with variation of large, small and average range are adjusted and initialized.

5. Using Triangular Function set up Output parameter as result with Range of large, small and average.

6. Generate the various Rules based on Input Parameters and by getting the Threshold Values set the Handoff Factor

7. Number of Nodes/Users are given Input as the $1,2 \ldots$ up to $\mathrm{N}$.

8. The Threshold Values of the input are given by the Step 6 such as bi, RSSi and Ci

9. The other networks detail and Current Connected Network are entered.

10. The Network with Input Values is trained

11. Get Historical Information and Perform Decision based on the Network Conditions, If Exist. Exit.

12. Otherwise Perform Decision Function and Allocate the Network, Saved for Future Training.

13. Exit 


\section{Execution Flow Chart}

A flowchart is a sort of figure that gives an algorithm, process or workflow presenting the steps as various kinds of boxes, and their sequence by linking them with arrows. This pictorial demonstration shows a solution model to a given problem.

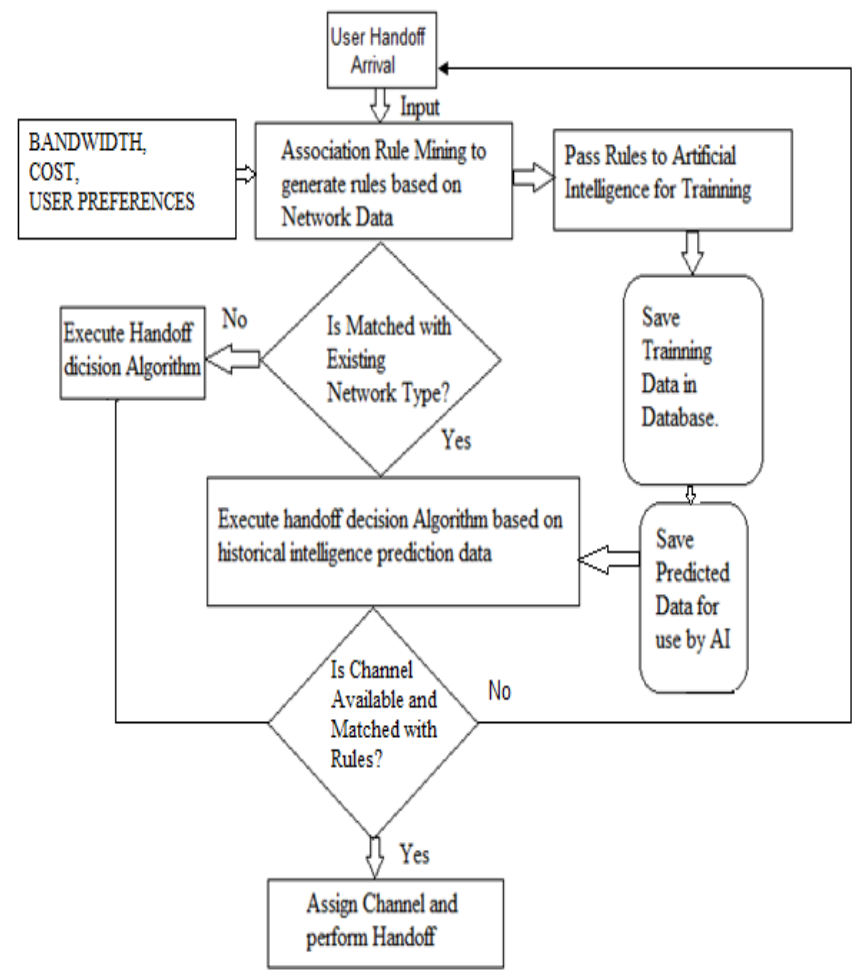

Handoff Decision Work Flow

\section{Fuzzy Rules Set}

The different sets of rules are defined with the various combinations such as Bandwidth Signal Strength, and Time. Depending upon the one value and the other, rules would vary. The table shows the combinations of these rules which can be used in the Fuzzy Logic editor.

Table 1: Rules Definitions

\begin{tabular}{|l|l|l|l|}
\hline Rules & RSS & BW & Time \\
\hline I & Small & Small & Small \\
\hline II & Small & Small & Average \\
\hline III & Small & Small & Large \\
\hline IV & Small & Average & Small \\
\hline V & Small & Average & Average \\
\hline VI & Small & Average & Large \\
\hline VII & Small & Large & Small \\
\hline VIII & Small & Large & Average \\
\hline IX & Small & Large & Large \\
\hline X & Average & Small & Small \\
\hline XI & Average & Small & Average \\
\hline XII & Average & Small & Large \\
\hline XIII & Average & Average & Small \\
\hline XIV & Average & Average & Average \\
\hline XV & Average & Average & Large \\
\hline XVI & Average & Large & Small \\
\hline XVII & Average & Large & Average \\
\hline XVIII & Average & Large & Large \\
\hline XIX & Large & Small & Small \\
\hline XX & Large & Small & Average \\
\hline XXI & Large & Small & Large \\
\hline XXII & Large & Average & Small \\
\hline XXIII & Large & Average & Average \\
\hline XXIV & Large & Average & Large \\
\hline & & & \\
\hline
\end{tabular}


Extended Vertical Handoff Decision in Next Generation Wireless Networks

\begin{tabular}{|l|l|l|l|}
\hline XXV & Large & Large & Small \\
\hline XXVI & Large & Large & Average \\
\hline XXVII & Large & Large & Large \\
\hline
\end{tabular}

\section{Result Analysis on the Proposed System}

The following table shows the result of Handoff Decision Delay with Historical and without Historical Method. The Results are repeatable and reliable.

\begin{tabular}{|l|l|l|}
\hline Experiment Sr. No. & Without Historical & With Historical \\
\hline 1 & 0.1322 & 0.0016 \\
\hline 2 & 1.976698 & 0.016 \\
\hline 3 & 1.9936 & 0.012 \\
\hline
\end{tabular}

Results of Handoff Delay

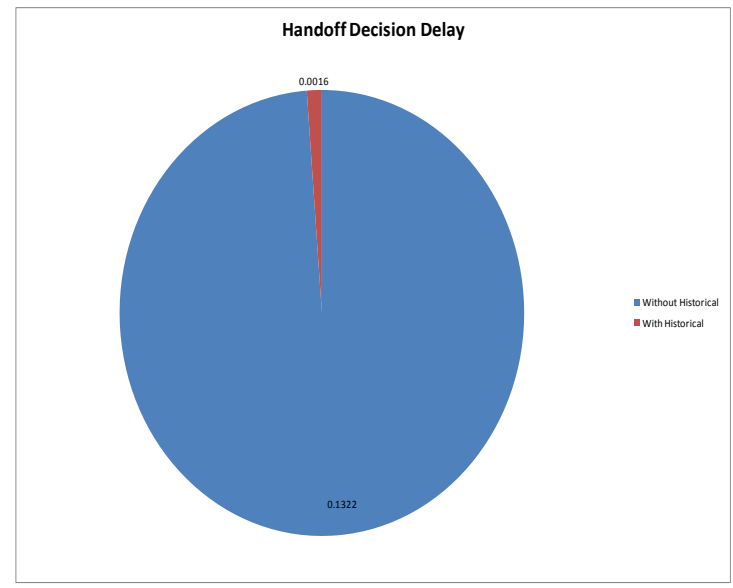

Figure: Experiment 1

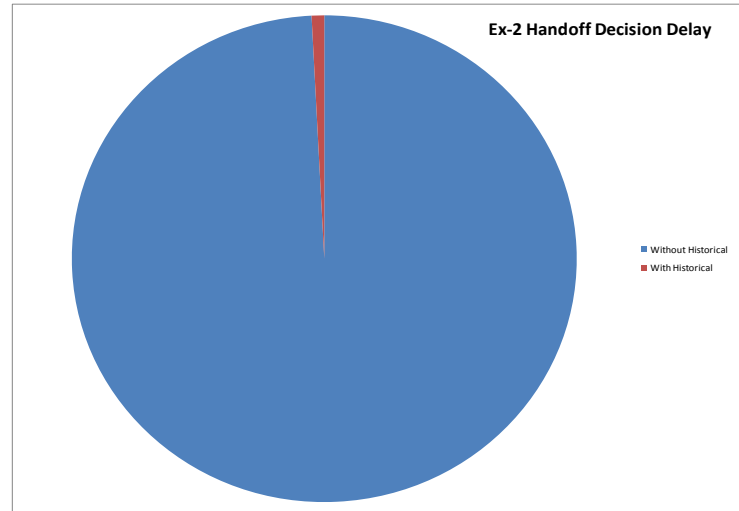

Figure: Experiment 2

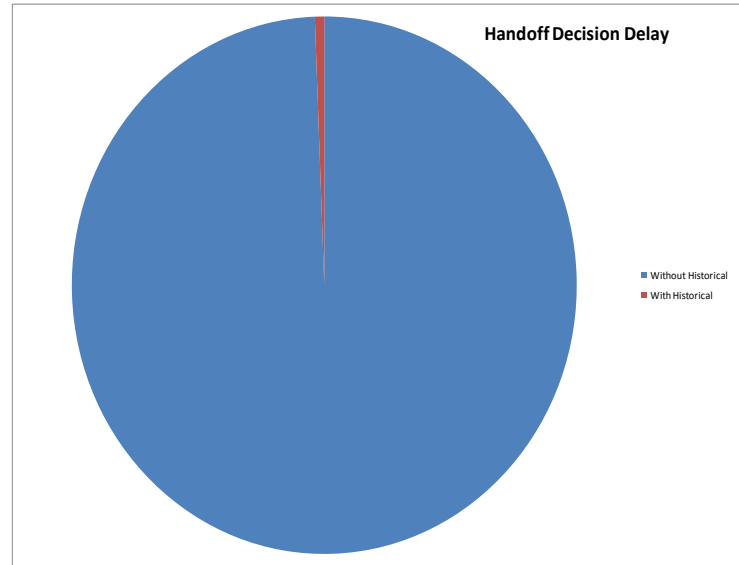

Figure: Experiment 3 
Analysis of Result: In this research extended historical vertical handoff decision function is developed. The results are compared and drawn as given in Table. The results show that handoff decision delay is reduced up to $99 \%$ with this scheme. The results are repeatable and reliable.

\section{Conclusion}

During this research, a scheme of vertical handoff in mobile communication environment has been presented. The existing system had the unnecessary handoffs which had been reduced in the proposed scheme. The main importance of the research work is in developing an efficient mechanism for vertical handover in wireless networks to reduce unnecessary handoffs and reduce the time delay based on the historical information and the rules generation using fuzzy system. Numbers of handoffs are reduced, which reduces the degradation in quality of signal and reduces extra load of network. Handoff latency is minimized. The algorithm has less computational complexity and is simple.

\section{Bibliography}

[1]. A. Bhuvaneswari, “ An Overview of Vertical Handoff Decision Making Algorithms” I. J. Computer Network and Information Security, 2012, 9, 55-62 Published Online August 2012 in MECS DOI: 10.5815/ijcnis.2012.09.07

[2]. A. J. Onumanyi and E. N. Onwuka, "Techniques for vertical handoff decision across wireless heterogeneous networks: A survey" Scientific Research and Essays Vol. 6(4), pp. 683-687, 18 February, 2011

[3]. A.Ferdinand Christopher, Dr.M.K.Jeyakumar ,"User Data Rate Based Vertical Handoff in 4g Wireless Networks", Journal of Theoretical and Applied Information Technology, ISSN: 1992-8645,2013

[4]. Abdelhaleem Saeed, Hafizal Mohamad, Mazlan Abbas, Borhanuddin Mohd Ali, “ WiFi/WiMAX Seamless Convergence with Adaptive Vertical Handover for Continuity of Internet Access" Advances in Internet of Things, 2011, 1, 32-37 doi:10.4236/ait.2011.12005 Published Online July 2011

[5]. Abdellatif Ezzouhairi , "Adaptive End-To-End Mobility Scheme for Seamless Horizontal and Vertical Handoffs", Ubiquitous Computing and Communication Journal.

[6]. Abhijit Bijwe Dr. C.G.Dethe, “Vertical Handoff algorithms using Neural networks” ISBN: 978-981-07-6260-5 doi:10.3850/ 978981-07-6260-5-10

[7]. Ahmed Hasswa, Nidal Nasser, Hossam Hassanein, "Generic Vertical Handoff Decision Function for Heterogeneous Wireless Networks" IEEE,2005

[8]. Ali A. Mohammed Gang Su, Dheyaa J. Kadhim,"A New Proposed Handoff Scheme for Mobile Communication Systems", IEEE,2011.

[9]. Bijay Paikaray, “Vertical Handoff Decision Based On Genetic Algorithm in 4G Network”

[10]. Chi-Yuan Chang, Hong-Jie Wang and Han-Chieh Chao, "Using Fuzzy Logic to Mitigate IEEE 802.11 Handoff Latency"

[11]. Dawei Mu; Xianlei Ge; Rong Chai,"Vertical Handoff Modeling and Simulation in VANET Scenarios",2013.

[12]. Dong Ma , "A QoS-Based Vertical Handoff Scheme for Interworking of WLAN and WiMAX”, IEEE. 2009

[13]. Enrique Stevens-Navarro, "Comparison between Vertical Handoff Decision Algorithms for Heterogeneous Wireless Networks"

[14]. G.S. Tomar ,'New Handoff Initiation Algorithm for Cellular Mobile Communication”, IEEE,2009

[15]. Iyad Alkhayat, Anup Kumar ,"End-to-End Mobility Solution for Vertical Handoff between Heterogeneous Wireless Networks", IEEE,2009

[16]. K.Savitha, Dr.C.Chandrasekar, “An Overview of Vertical Handoff Decision Based On MADM For Heterogeneous Wireless Network", Journal of Computer Applications, Vol-III, No.3, July - Sept 2010.

[17]. M. Z. A. Syuhada, I. Mahamod,'Performance Evaluation of Vertical Handoff in Fourth Generation (4G) Networks Model", IEEE,2008.

[18]. Mandeep Kaur Gondara and Dr. Sanjay Kadam, " Requriments of Vertical Handoff Mechanism in 4G Wireless Networks" International Journal of Wireless \& Mobile Networks (IJWMN) Vol. 3, No. 2, April 2011

[19]. Meriem Kassar , Brigitte Kervella, "An overview of vertical handover decision strategies in heterogeneous wireless networks" ELSEVIER Computer Communications 2008 2607-2620

[20]. R.Tawil \& Guy Pujolle, "A Vertical Handoff Decision Scheme in Heterogeneous Wireless Systems”, IEEE, 2008 